\title{
Efeitos do genótipo e da condição corporal sobre o desempenho de cordeiros terminados em confinamento ${ }^{1}$
}

\section{Felipe Queiroga Cartaxo ${ }^{2}$, Wandrick Hauss de Sousa ${ }^{3}$, Marcílio Fontes Cezar ${ }^{4}$, Severino Gonzaga Neto ${ }^{5}$, Maria das Graças Gomes Cunha ${ }^{3}$}

\footnotetext{
1 Projeto financiado pelo convênio FINEP/EMEPA-PB/FAPESQ.

2 Programa de Doutorado Integrado em Zootecnia/UFPB - Areia-PB.

${ }^{3}$ EMEPA-PB

${ }^{4}$ Departamento de Medicina Veterinária/CSTR/UFCG - Patos-PB.

${ }^{5}$ Departamento de Zootecnia/CCA/UFPB - Areia-PB.
}

RESUMO - Avaliaram-se o desempenho e a margem bruta de lucro de cordeiros terminados em confinamento. Foram utilizados 32 cordeiros não-castrados - 16 Santa Inês (SI) e 16 1⁄2 Dorper × 1/2 Santa Inês (DpSI) - com 103 dias de idade e $20,0 \mathrm{~kg}$ de peso corporal. Os cordeiros receberam ração completa com 30\% de feno de maniçoba (Manihot pseudoglaziovii) e $70 \%$ de concentrado. O período de adaptação às instalações e à dieta foi de 14 dias. O delineamento experimental foi inteiramente casualizado em esquema fatorial $2 \times 2$, composto de dois genótipos e dois escores corporais. Não foi observado efeito significativo dos genótipos sobre nenhuma característica de desempenho estudada, contudo, os mestiços (DpSI) possibilitaram maior margem bruta de lucro. A condição corporal afetou significativamente o consumo de matéria seca, a conversão alimentar e o número de dias em confinamento. Os cordeiros com condição corporal intermediária consumiram $1,04 \mathrm{~kg} / \mathrm{dia}$ e $82,64 \mathrm{~g} / \mathrm{kg}^{0,75}$, enquanto aqueles com condição gorda consumiram 1,15 kg/dia e 86,79 g/kg0,75. Os cordeiros com condição corporal intermediária apresentaram menor consumo de matéria seca e melhor conversão alimentar, o que resultou em menor número de dias em confinamento e maior margem bruta de lucro em comparação aos cordeiros com condição corporal gorda, independentemente do genótipo.

Palavras-chave: consumo, conversão alimentar, cruzamento, Dorper, escore corporal, Santa Inês

\section{Effects of genotype group and of the body condition on the performance of lambs finished in feedlot}

\begin{abstract}
The objective of this study was to evaluate the performance and the gross margin of profit of lambs finished in feedlot. Thirty-two no castrate male lambs from the genotype Santa Inês and crossbred Dorper $\times$ Santa Inês and two body condition, intermediate and fat, were used. The lambs were 16 Santa Inês (SI) and $161 / 2$ Dorper $\times 1 / 2$ Santa Inês (DpSI), with average 103 days old and $20.0 \mathrm{~kg} \mathrm{BW}$. The lambs were full fed ration with 30\% of maniçoba hay (Manihot pseudoglaziovii) and $70 \%$ of concentrate. The adaptation period to the installation and the diet was of 14 days. A completely randomized design in a $2 \times 2$ factorial arrangement (genotype and body conditions score) was used. There was no significant effect of genotypes on any performance characteristic, however, the crossbred (DpSI) obtained greater margin of profit. The body condition affected the dry matter intake, feed conversion and the number of days in feedlot. The lambs with intermediate body condition consumed $1.04 \mathrm{~kg} /$ day and $82.64 \mathrm{~g} / \mathrm{kg}{ }^{0.75}$, while those with fat body condition consumed 1.15 $\mathrm{kg} /$ day and $86.79 \mathrm{~g} / \mathrm{kg}^{0.75}$. The lambs with intermediate body condition showed lower dry matter intake and better feed conversion, which resulted in fewer days in feedlot and greater gross margin of profit compared to lambs with fat body condition, independently of the genotype.
\end{abstract}

Key Words: body score, crossbred, Dorper, feeding efficiency, intake, Santa Inês

\section{Introdução}

A ovinocultura tem grande importância no contexto socioeconômico da Região Nordeste do Brasil, pois gera oportunidades de emprego e renda. Essa região detém efetivo ovino representativo, com aproximadamente 56,5\% do efetivo nacional (IBGE, 2003). A estacionalidade da produção de forragem, no entanto, é um problema para a produção pecuária do Brasil, pois existem períodos de escassez e com baixo valor nutritivo e períodos de abundância com valor nutritivo relativamente alto (Gonzaga Neto et al., 2001; Rodrigues et al., 2003; Araújo et al., 2004; Véras et al., 2005b). 
A terminação de cordeiros em confinamento para abate pode ser uma alternativa zootecnicamente viável e eficiente para produção de carne ovina de qualidade, pois resulta em regularidade na oferta, além de padronização das carcaças. Outra vantagem do confinamento de cordeiros é a baixa mortalidade dos animais, em razão do maior controle sanitário e nutricional, que resulta em abate precoce e carcaças de qualidade (Barreto et al., 2004).

O cruzamento de raças explorando a heterose, principalmente utilizando-se raças especializadas para produção de carne, tem sido preconizado por vários autores como forma de aumentar a produção de carne (Muniz et al., 1997; Garcia et al., 2003). Segundo Sousa et al. (2003), uma das alternativas para melhorar o desempenho e as características de carcaça de cordeiros Santa Inês é o cruzamento com ovinos da raça Dorper.

Considerando o rebanho de fêmeas na Região Nordeste e a alta rusticidade e adaptabilidade, a raça Santa Inês poderia ser utilizada como raça materna em cruzamentos com a raça Dorper, o que melhoraria o desempenho e as características de carcaças dos cordeiros resultantes desse cruzamento.

Neste sentido, Furusho-Garcia et al. (2004) concluíram que o uso de raça especializada para produção de carne no cruzamento com ovelhas da raça Santa Inês melhorou o desempenho dos animais. Snowder \& Duckeet (2003), avaliando cordeiros mestiços de Dorper, observaram ganho de peso diário de 283 g do nascimento aos 118 dias de idade.

A determinação do ponto ótimo de acabamento de cordeiros por meio da avaliação do escore corporal pode reduzir o período de terminação e o custo dos cordeiros no confinamento. Segundo Osório \& Osório (2003), o escore corporal é uma medida subjetiva, que, por meio da palpação da região lombar ou da inserção da cauda do animal, permite estimar a relação músculo/gordura. O escore corporal indica a quantidade de reserva de gordura e de sua distribuição no corpo do animal. Oregui et al. (1991) concluíram em experimento com ovelhas Latxa que cada ponto de aumento na escala do escore corporal equivale a aproximadamente $5 \mathrm{~kg}$ ou 9,8\% de peso corporal.

A alimentação aumenta o custo de produção de cordeiros em confinamento (Zundt et al. 2002). Para o confinamento de cordeiros se tornar uma opção economicamente viável, é necessário utilizar dieta de baixo custo, que proporcione alto ganho de peso médio diário e boa conversão alimentar para reduzir o período no confinamento e aumentar a margem de lucro.

Almeida Jr. et al. (2004) observaram custo médio com alimentação em cordeiros até atingirem o peso de abate de
R\$ 8,14. Siqueira et al. (2001) avaliando cordeiros nãocastrados e cordeiras Corriedale abatidos com pesos diferentes, encontraram renda líquida de $\mathrm{R}$ \$-6,17 a R \$ 17,73 por animal.

Objetivou-se com este estudo avaliar o desempenho animal e a margem bruta de lucro de cordeiros Santa Inês (SI) e mestiços $1 \frac{1}{2}$ Dorper $\times 1 \frac{1}{2}$ Santa Inês $(\mathrm{DpSI})$ terminados em confinamento com diferentes condições corporais.

\section{Material e Métodos}

O experimento foi conduzido na Estação Experimental Pendência, base física da Empresa Estadual de Pesquisa Agropecuária da Paraíba (EMEPA-PB), localizada no município de Soledade, Paraíba, mesorregião do Cariri, situada nas coordenadas geográficas com latitude de $7^{0} 8^{\prime} 18^{\prime \prime} \mathrm{S}$ e $36^{\circ} 27^{\prime} 2^{\prime \prime} \mathrm{W}$, com altitude de $534 \mathrm{~m}$ e temperatura média de $30^{\circ} \mathrm{C}$.

Foram utilizados 32 cordeiros não-castrados - 16 da raça Santa Inês (SI) e 16 mestiços $1 \frac{1}{2}$ Dorper $\times 1 / 2$ Santa Inês (DpSI) - com 103 dias de idade e peso médio de 20,0 kg. Os animais foram inicialmente vacinados contra clostridioses e vermifugados via oral com cloridrato de levamisol 7,5\%. Após 15 dias, procedeu-se a nova vermifugação com aplicação subcutânea de ivermectina 1\%.

Os animais foram distribuídos em baias individuais medindo $0,80 \times 1,20 \mathrm{~m}$, todas com acesso livre aos comedouros e bebedouros, onde permaneceram em regime de confinamento até atingirem a condição corporal preconizada para o abate. O período de adaptação foi de 14 dias e as pesagens dos cordeiros foram realizadas a cada 14 dias. Utilizou-se dieta única, com relação volumoso:concentrado 30:70, com $16,0 \%$ de proteína bruta, 3,60\% de extrato etéreo, $28,0 \%$ de fibra em detergente neutro, 4,18\% de matéria mineral e 2,70 Mcal de energia metabolizável por quilograma de matéria seca - MS (Tabela 1).

Estabeleceu-se um consumo de MS de 5\% do peso corporal (PC), reajustado e pesado diariamente de acordo com a quantidade de sobras (10\%), para posterior cálculo dos consumos de MS (CMS).

A avaliação do escore corporal foi feita por três examinadores, que classificaram os cordeiros em duas condições corporais: intermediária (escore 2,5 a 3,5) e gorda (escore 4,0 a 5,0), com adaptação à metodologia descrita por Osório \& Osório (2003). Para a atribuição dos escores, foram feitas avaliações semanais por meio de exame visual e palpação da região lombar, esternal e na inserção da cauda dos cordeiros .

O critério para realização dos abates foi a condição corporal: quando os cordeiros de cada genótipo atingiram 
Tabela 1 - Composição das dietas experimentais

\begin{tabular}{lccccccc}
\hline Ingrediente & MS (\%) & PB (\%) & EE (\%) & EM (Mcal/kg de MS) & FDN (\%) & MM (\%) & Dieta (\%) \\
\hline Feno de maniçoba & 83,7 & 11,6 & 3,44 & 1,95 & 58,00 & 6,94 & 30,0 \\
Milho moído & 89,0 & 9,0 & 4,60 & 3,15 & 15,76 & 1,45 & 47,0 \\
Farelo de soja & 89,0 & 46,0 & 1,10 & 3,18 & 15,74 & 7,11 & 16,5 \\
Farelo de trigo & 88,0 & 17,1 & 4,40 & 2,70 & 31,00 & 5,40 & 4,0 \\
Calcário & 99,0 & - & - & - & - & 100,0 & 1,5 \\
Sal mineral & 99,0 & - & - & - & - & 100,0 & 1,0 \\
\hline
\end{tabular}

a condição corporal intermediária, realizou-se o primeiro abate; e, quando os cordeiros atingiram a condição corporal gorda, realizou-se o segundo abate. Antes, foram pesados para cálculo do ganho de peso médio diário (GPMD) e da conversão alimentar (CA).

A margem bruta foi calculada considerando o ganho de peso total no período de confinamento, o consumo total de MS e as despesas com vacina e medicamentos. Os cálculos foram realizados com adaptação da metodologia descrita por Véras et al. (2005a) e a margem bruta de lucro foi determinada utilizando-se a fórmula:

$$
\mathrm{MB}=\mathrm{GPT} \times 6,41-(\mathrm{CTD} \times 1,20)-\mathrm{DVM}
$$

em que: $\mathrm{MB}=$ margem bruta de lucro (U\$/animal); $\mathrm{GPT}=$ ganho de peso total; 6,41= preço por kg vivo de cordeiro na região (U\$); CTD = consumo total da dieta $(\mathrm{kg}) ; 1,22$ = preço por $\mathrm{kg}$ de MS (U\$); DVM = despesas com vacina e medicamento(U\$).

Avaliaram-se o consumo de MS (CMS), expresso em quilograma por dia $(\mathrm{kg} / \mathrm{dia})$, em gramas por unidade de tamanho metabólico ( $\mathrm{g} / \mathrm{kg}^{0,75}$ ) e em porcentagem do peso corporal (\% PC); o ganho de peso médio diário (GPMD), em g/dia; a conversão alimentar (CA); o número de dias em confinamento (DC); e a margem bruta (MB), em U\$/animal.

Os dados foram submetidos a análise de variância em delineamento inteiramente casualizado em esquema fatorial $2 \times 2$ (dois genótipos e dois escores corporais) utilizando-se o teste $\mathrm{F}$ a $5 \%$ de probabilidade para comparação dos quadrados médios dos fatores testados.

O modelo matemático utilizado foi o seguinte:

$$
Y_{i j}=\mu+G_{i}+E_{j}+G_{i j}+\varepsilon_{i j} \text {, }
$$

em que $\mathrm{Y}_{\mathrm{ij}}=$ valor da variável dependente estudada, $\mu=$ média geral; $\mathrm{G}_{\mathrm{i}}=$ efeito do genótipo $\mathrm{i} ; \mathrm{E}_{\mathrm{j}}=$ efeito do escore $\mathrm{j} ; \mathrm{GE}_{\mathrm{ij}}=$ interação genótipo $\times$ escore; e $\varepsilon_{\mathrm{ij}}=$ erro aleatório associado a cada observação.

\section{Resultados e Discussão}

Não foi observado efeito significativo da interação genótipo $\times$ condição corporal sobre as características de desempenho estudadas (Tabela 2). Os genótipos não influenciaram ( $\mathrm{P}>0,05)$ os consumos de MS, cujos valores foram de 1,12 kg e 1,06 kg, respectivamente, para os cordeiros Santa Inês e mestiços. Esses resultados estão de acordo com o preconizado pelo NRC (1985), 1,0 a 1,3 kg/dia, para esta catergoria animal. Esse alto consumo pode ser atribuído ao teor de fibra em detergente neutro (28\%) da dieta, que, quando é superior a 50\%, limita a ingestão de MS, em virtude da capacidade física do rúmen (Mertens, 1994). Quando é inferior a 28\%, pode ocasionar redução no consumo, em razão da densidade energética da dieta.

Valores inferiores ( $0,88 \mathrm{~kg}$ de MS/dia) aos encontrados neste estudo foram observados por Alves et al. (2003) em cordeiros Santa Inês com peso inicial de 20,0 kg alimentados com dietas com diversos níveis de energia. Valores próximos aos obtidos neste estudo foram observados por Véras et al. (2005a), que, ao testarem diferentes níveis de substituição do milho por farelo de palma na alimentação de cordeiros mestiços pesando 20,0 kg, registraram consumo de 1,10 a $1,19 \mathrm{~kg} / \mathrm{dia}$. Clementino (2004), em pesquisa com cordeiros $1 / 2$ Dorper $\times 1 / 2$ Santa Inês em confinamento recebendo diferentes níveis de concentrado, observou consumo de MS de 1,0 a 1,19 kg/dia. Resultados de consumos superiores, no entanto, de aproximadamente 1,25 kg de MS/dia, foram encontrados por Neiva et al. (2004), em experimento com cordeiros Santa Inês não-castrados com 19,0 kg PC alimentados com dieta com mesma relação volumoso:concentrado utilizada neste estudo.

Diferentemente dos resultados de consumo de MS obtidos neste estudo, Sá et al. (2005), avaliando cordeiros Santa Inês e Hampshire Down em confinamento alimentados com dieta contendo 16\% de PB e 75\% de NDT, observaram diferença $(\mathrm{P}<0,05)$ no consumo de MS entre os genótipos. O consumo de MS por unidade de tamanho metabólico também não diferiu $(\mathrm{P}>0,05)$ entre os genótipos; as médias obtidas para os cordeiros Santa Inês foram de $85,48 \mathrm{~g} / \mathrm{kg}^{0,75}$ e para os mestiços (DpSI), de 83,01 g/ $\mathrm{kg}^{0,75}$. O NRC (1985) estabelece para cordeiros com ganho de peso de $300 \mathrm{~g} / \mathrm{dia}$ consumo de MS de $100 \mathrm{~g} / \mathrm{kg}^{0,75}$, no entanto, o valor obtido neste estudo foi menor, o que comprova discrepância entre 
Tabela 2 - Consumo de matéria seca (CMS), ganho de peso médio diário (GPMD), conversão alimentar (CA) e dias de confinamento (DC) de cordeiros de dois grupos genéticos abatidos com duas condições corporais

\begin{tabular}{|c|c|c|c|c|}
\hline \multirow[t]{2}{*}{ Variável } & \multicolumn{2}{|c|}{ Genótipo } & \multicolumn{2}{|c|}{ Condição corporal } \\
\hline & SI & DpSI & Intermediária & Gorda \\
\hline № de observações & 16 & 16 & 19 & 13 \\
\hline Peso vivo inicial (kg) & 20,4 & 19,2 & 19,7 & 19,9 \\
\hline Peso vivo final $(\mathrm{kg})$ & 30,6 & 29,5 & 29,1 & 31,4 \\
\hline Consumo de MS (kg/dia) & $1,12 \mathrm{a}$ & $1,06 \mathrm{a}$ & $1,04 \mathrm{a}$ & $1,15 b$ \\
\hline Consumo de MS (g/kg $\left.{ }^{0,75}\right)$ & $85,48 a$ & $83,17 \mathrm{a}$ & $82,64 a$ & $86,79 b$ \\
\hline Consumo de MS (\%PC) & $3,63 a$ & $3,57 a$ & $3,56 a$ & $3,67 \mathrm{a}$ \\
\hline Ganho de peso médio diário (g/animal.dia) & $281,0 \mathrm{a}$ & $291,0 \mathrm{a}$ & $296,0 a$ & $273,0 \mathrm{a}$ \\
\hline Conversão alimentar & $4,06 \mathrm{a}$ & $3,69 a$ & $3,62 \mathrm{a}$ & $4,24 b$ \\
\hline Dias de confinamento & $36,75 a$ & $35,87 a$ & $32,42 \mathrm{a}$ & $42,0 \mathrm{~b}$ \\
\hline
\end{tabular}

Médias seguidas de distintas nas linhas diferem $(P<0,05)$ pelo teste $F$ a $5 \%$ de probabilidade.

SI = Santa Inês; DpSI = 1/2 Dorper $+1 / 2$ Santa Inês

Condição corporal: intermediária (escore corporal 2,5 a 3,5); gorda (escore corporal 4,0 a 5,0).

as exigências dos animais criados no Nordeste do Brasil e as recomendações dos boletins internacionais de nutrição animal.

Os valores encontrados neste estudo foram superiores aos observados por Oliveira et al. (2003), de 70,60 g/ $\mathrm{kg}^{0,75}$, em cordeiros Santa Inês alimentados com dietas isoprotéicas com 15,0\% de PB. Do mesmo modo, Camurça et al. (2002) observaram consumo de 74,28 g/ $\mathrm{kg}^{0,75}$. Os resultados deste estudo foram inferiores, no entanto, aos 88,12 a 90,58 g/ $\mathrm{kg}^{0,75}$ observados por Barreto et al. (2004) em cordeiros Santa Inês alimentados com dietas contendo dejeto de suínos.

Os consumos de MS calculados em porcentagem do PC foram semelhantes entre os genótipos (SI 3,63\% e DpSI 3,57\%), mas estiveram abaixo do sugerido pelo NRC (1985), de 4,3 a 5,0\% PC para cordeiros de 20 a $30 \mathrm{~kg}$. Valores inferiores foram obtidos por Alves et al. (2003), de 3,30\% PC. Consumos de MS superiores aos verificados neste estudo foram observados por Véras et al. (2005a), 4,11 a 4,36\% PC, em cordeiros em confinamento alimentados com dietas contendo farelo de palma. Segundo esses autores, este alto consumo de MS esteve relacionado à alta palatabilidade da palma forrageira e à elevada taxa de digestão ruminal desse alimento.

$\mathrm{O}$ ganho de peso médio não diferiu $(\mathrm{P}>0,05)$ entre os genótipos: 281 g/dia para os cordeiros Santa Inês e $291 \mathrm{~g} /$ dia para os mestiços (DpSI). Este alto ganho de peso diário pode ser explicado pela composição da dieta e pelo potencial de ganho dos cordeiros, principalmente os Santa Inês, uma vez que todos os animais eram Prov I ou II (cordeiros Puros por Cruza oriundos de acasalamentos com ovelhas Santa Inês BASE [fêmeas Puras por Cruza de Origem Desconhecida] ou de ovelhas Santa Inês Puras por Cruza de Origem Conhecida com reprodutores Puros de
Origem Santa Inês). A primeira geração recebe o Registro Genealógico com denominação Prov I (provisório 1), a segunda, Prov II e a terceira, Prov III. Os animais da quarta geração são considerados Puros de Origem.

A dieta utilizada neste estudo continha 2,70 Mcal de energia metabolizável/kg de MS, valor próximo à exigência estabelecida pelo NRC (1985), de 2,80 Mcal de energia metabolizável $/ \mathrm{kg}$ de MS para cordeiros nesta categoria com ganho de $300 \mathrm{~g} / \mathrm{dia}$.

Entretanto, Vasconcelos et al. (2000), avaliando cordeiros Santa Inês com peso inicial de 32,10 kg alimentados com $4 \mathrm{~kg}$ de volumoso e $0,50 \mathrm{~kg}$ de concentrado com $20 \%$ $\mathrm{PB}$, verificaram ganho de peso diário mais baixo (252 g/ animal). Resultados inferiores também foram reportados por Freitas et al. (2005), 130 a 211 g, em pesquisa com cordeiros mestiços Santa Inês em confinamento com diferentes idades de desmame.

Furusho-Garcia et al. (2000), em experimento com ovinos Santa Inês, Santa Inês × Texel e Bergamácia × Texel alimentados com dietas contendo casca de café, não observaram diferenças $(\mathrm{P}>0,05)$ no ganho de peso diário. Resultados semelhantes foram verificados por Sá et al. (2005) em cordeiros Santa Inês e Hampshire Down.

A conversão alimentar foi semelhante $(\mathrm{P}>0,05)$ entre genótipos (4,06 para SI e de 3,69 para DpSI). Esse bom resultado pode ser atribuído à relação volumoso:concentrado (30:70) e ao nível nutricional da dieta. Valores inferiores foram descritos por Alves et al. (2003) em estudo com cordeiros Santa Inês e por Clementino (2004) em pesquisa com cordeiros $1 / 2$ Dorper $\times 1 \frac{1}{2}$ Santa Inês. Resultados próximos aos deste estudo foram relatados por Susin et al. (2000) em cordeiros Santa Inês alimentados com dieta com 20\% de volumoso e $80 \%$ de concentrado. Esses autores encontraram médias de 3,57 a 3,89, enquanto Yamamoto et al. (2005), 
avaliando cordeiros Santa Inês puros e mestiços Dorset $\times$ Santa Inês, obtiveram valores de 3,82 e 3,64, respectivamente.

Os resultados obtidos corroboram os de FurushoGarcia et al. (2000), que avaliaram o efeito do genótipo sobre a conversão alimentar de cordeiros Santa Inês, Santa Inês $\times$ Texel e Bergamácia $\times$ Texel. Neste estudo não houve influência significativa $(\mathrm{P}>0,05)$ dos genótipos sobre $\mathrm{o}$ número de dias em confinamento. As médias observadas foram de 36,75 dias para os cordeiros Santa Inês e 35,87 dias para os DpSI, portanto, os genótipos estudados apresentaram resultados similares de deposição de tecido na carcaça.

No cálculo da margem bruta de lucro (U\$/animal), de acordo com genótipo e condição corporal, foram considerados o ganho de peso total por animal no período de confinamento, os custos com a dieta e as despesas com vacina e medicamentos (Tabela 3). A margem bruta de lucro foi maior nos mestiços de Dorper (U\$ 18,62/animal) em relação aos Santa Inês (U\$ 14,20/animal), provavelmente em virtude do menor consumo e da melhor conversão alimentar.

Houve efeito significativo $(\mathrm{P}<0,05)$ da condição corporal sobre o consumo de MS em $\mathrm{kg} / \mathrm{dia}$ e $\mathrm{g} / \mathrm{kg}^{0,75}$. Cordeiros abatidos com a condição corporal intermediária apresentaram menor consumo de MS em kg/dia e g/kg0,75 em comparação aos abatidos com a condição corporal gorda. Esse resultado pode ser justificado pelo aumento do peso corporal dos cordeiros, que aumenta simultaneamente a quantidade de ração ofertada e ingerida.

O consumo de MS não foi influenciado $(\mathrm{P}>0,05)$ pelo percentual do peso corporal. Como as quantidades da dieta eram ajustadas diariamente e o ganho de peso também foi diário, provavelmente houve sincronia da dieta consumida com o aumento do percentual do peso corporal dos cordeiros estudados.

Para o ganho médio de peso diário, houve semelhança $(P>0,05)$ entre as condições corporais avaliadas, de modo que, para a condição intermediária, o ganho foi de 296 g, enquanto, para a condição gorda, foi de 273 g. Souza et al. (2004), em pesquisa com cordeiros mestiços Ile de France $\times$ Corriedale não-castrados alimentados com dieta isoproteíca (16,5\%) e isocalórica (67,6\% NDT), não encontraram efeito significativo $(\mathrm{P}>0,05)$ da condição corporal sobre o ganho médio de peso diário.

A condição corporal influenciou significativamente $(\mathrm{P}<0,05)$ a conversão alimentar, provavelmente em virtude do maior desenvolvimento de músculos e de gorduras, que implica a formação de gorduras e maior quantidade de ração consumida, elevando a conversão alimentar e resultando nas médias de 3,62 para a condição corporal intermediária e de 4,24 para a condição corporal gorda.

Na literatura revisada, não foram encontrados trabalhos relacionando o efeito da condição corporal sobre o desempenho de animais, portanto, não foi possível fazer comparações para os consumos de MS e a conversão alimentar. O número de dias em confinamento diferiu $(\mathrm{P}<0,05)$ entre cordeiros com diferentes condições corporais. Os cordeiros abatidos com condição corporal intermediária foram mantidos por menos tempo em confinamento (aproximadamente 10 dias) em relação àqueles abatidos com condição corporal gorda, o que reduziu a quantidade de ração e proporcionou maior giro de capital.

Observou-se maior margem bruta de lucro para os cordeiros abatidos com condição corporal intermediária (Tabela 3), resultado relacionado ao menor consumo total da dieta e à melhor conversão alimentar dos animais nesta condição corporal. Siqueira et al. (2001), avaliando os resultados econômicos de cordeiros confinados, concluíram que os machos mantidos por menor tempo no confinamento apresentaram melhor conversão alimentar e maior renda líquida/animal.

Na condição corporal intermediária, o cordeiro tem sua cobertura muscular na apófise espinhosa dorsal e lombar

Tabela 3 - Margem bruta (U\$/animal) de cordeiros de dois grupos genéticos abatidos com duas condições corporais

\begin{tabular}{|c|c|c|c|c|}
\hline \multirow[t]{2}{*}{ Variável } & \multicolumn{2}{|c|}{ Genótipo } & \multicolumn{2}{|c|}{ Condição corporal } \\
\hline & SI & DpSI & Intermediária & Gorda \\
\hline $\mathrm{N}^{0}$ de observações & 16 & 16 & 19 & 13 \\
\hline Peso corporal inicial (kg) & 20,4 & 19,2 & 19,7 & 19,9 \\
\hline Peso corporal final (kg) & 30,6 & 29,5 & 29,1 & 31,4 \\
\hline Dieta (kg de MS (U\$)) & 1,20 & 1,20 & 1,20 & 1,20 \\
\hline Preço inicial cordeiro (U\$) & 130,80 & 123,11 & 126,31 & 127,60 \\
\hline Consumo total da dieta/cordeiro (U\$) & 49,39 & 45,61 & 40,46 & 57,96 \\
\hline Despesas vacina e medicamentos (U\$) & 1,80 & 1,80 & 1,80 & 1,80 \\
\hline Preço final cordeiro (U\$) & 196,20 & 189,15 & 186,59 & 201,34 \\
\hline Margem bruta/cordeiro (U\$) & 14,20 & 18,62 & 18,00 & 13,97 \\
\hline
\end{tabular}

SI= Santa Inês; DpSI = 1/2 Dorper + 1/2 Santa Inês.

Condição corporal: intermediária (escore corporal 2,5 a 3,5); gorda (escore corporal 4,0 a 5,0).

$(\mathrm{U} \$ 1,00=\mathrm{R} \$ 2,29)$. 
ligeiramente coberta por tecido adiposo, portanto, os músculos estão bem definidos. Para atingir esta condição, é necessária quantidade menor de ração que para formação de tecido adiposo. No entanto, para atingir a condição corporal gorda, os cordeiros acumulam gordura em todas as regiões do corpo, assim, a demanda energética é bem maior para formação da gordura subcutânea e interna que para a formação de músculos. O custo por animal é maior para esta condição corporal, o que diminui, conseqüentemente, a margem bruta de lucro.

A melhor relação custo/benefício foi obtida para os cordeiros com condição corporal intermediária, que apresentaram margem bruta 28,8\% superior à obtida na condição corporal gorda. Os cordeiros nesta condição são abatidos com mais baixo peso, porém, o menor consumo total da dieta, a melhor conversão alimentar e o menor período para o acabamento dos animais, assim como a melhor relação custo/benefício, não justificam a espera por cordeiros com condição corporal gorda, que exigem maior tempo de retorno do capital.

\section{Conclusões}

Nas condições deste estudo, cordeiros Santa Inês e mestiços de Dorper apresentam desempenhos similares e satisfatórios, portanto, podem ser utilizados no confinamento com êxito. Cordeiros mestiços (DpSI) apresentaram maior margem bruta de lucro em comparação aos Santa Inês. Cordeiros abatidos com condição corporal intermediária apresentam menor consumo de MS, melhor conversão alimentar, menor número de dias em confinamento e maior margem bruta. A condição corporal intermediária pode ser preconizada como critério para abate de cordeiros.

\section{Literatura Citada}

ALMEIDA JR., G.A.; COSTA, C.; MONTEIRO, A.L.G. et al. Desempenho, características de carcaça e resultado econômico de cordeiros criados em creep feeding com silagem de grãos úmidos de milho. Revista Brasileira de Zootecnia, v.33, n.4, p.1048-1059, 2004.

ALVES, K.S.; CARVALHO, F.F.R.; VERÁS, A.S.C. et al. Níveis de energia em dietas para ovinos Santa Inês: desempenho. Revista Brasileira de Zootecnia, v.32, n.6, p.1937-1944, 2003. (supl.2).

ARAÚJO, G.G.L.; MOREIRA, J.N.; FERRREIRA, M.A. et al. Consumo voluntário e desempenho de ovinos submetidos a dietas contendo diferentes níveis de feno de maniçoba. Revista Ciência Agronômica, v.35, n.1, p.123-130, 2004.

BARRETO, C.M.; AZEVEDO, A.R.; SALES, R.O. et al. Desempenho de ovinos em terminação alimentados com dietas contendo diferentes níveis de dejetos de suínos. Revista Brasileira de Zootecnia, v.33, n.6, p.1858-1865, 2004. (supl.1).

CAMURCA, D.A.; NEIVA, J.N.M.; PIMENTEL, J.C.M. et al. Desempenho produtivo de ovinos alimentados com dietas à base de feno de gramíneas tropicais. Revista Brasileira de Zootecnia, v.31, n.5, p.2113-2122, 2002.

CLEMENTINO, R.H. Efeito bioeconômico dos diferentes níveis de concentrado na dieta de cordeiros terminados em confinamentos. Areia: Universidade Federal da Paraíba, 2004 51p. Dissertação (Mestrado em Zootecnia) - Universidade Federal da Paraíba, 2004

FREITAS, D.C.; OLIVEIRA, G.J.C.; JAEGER, S.M.P. et al. Idade de desmama de cordeiros deslanados para terminação em confinamento, no litoral norte da Bahia. Revista Brasileira de Zootecnia, v.34, n.4, p.1392-1399, 2005.

FURUSHO-GARCIA, I.F..; PEREZ, J.R.O.; TEIXEIRA, J.C. et al. Desempenho de cordeiros Texel x Bergamácia, Texel x Santa Inês e Santa Inês puros, terminados em confinamento, alimentados com casca de café como parte da dieta. Revista Brasileira de Zootecnia, v.29, n.2, p.564-572, 2000.

FURUSHO-GARCIA, I.F.; PEREZ, J.R.O.; BONAGURIO, S. et al. Desempenho de cordeiros Santa Inês puros e cruzas Santa Inês com Ile de France e Bergamácia. Revista Brasileira de Zootecnia, v.33, n.6, p.1591-1603, 2004.

GARCIA, C.A.; MONTEIRO, A.L.G.; COSTA, C. et al. Medidas objetivas e composição tecidual da carcaça de cordeiros alimentados com diferentes níveis de energia em creep feeding. Revista Brasileira de Zootecnia, v.32, n.6, p.13801390, 2003.

GONZAGA NETO, S; BATISTA, A.M.V.; CARVALHO, F.F.R. et al. Composição bromatológica, consumo e digestibilidade in vivo de dietas com diferentes níveis de feno de catingueira (Caesalpinea bracteosa), fornecidas para ovinos Morada Nova. Revista Brasileira de Zootecnia, v.30, n.2, p.553-562, 2001.

INSTITUTO BRASILEIRO DE GEOGRAFIA E ESTATÍSTICA IBGE. [2003]. Pesquisa Pecuária Municipal. Disponível em: <http://www.ibge.gov.br> Acesso em: 16/03/2005.

MERTENS, D.R. Regulation of forage intake. In: FAHEY JR., G.C. (Ed.) Forage quality, evaluation and utilization. Madison: American Society of Agronomy, 1994. p.450-493.

MUNIZ, E.N.; PIRES, C.C.; SILVA, J.H. et al. Crescimento ponderal e características da carcaça de cordeiros de diferentes genótipos. In: REUNIÃO ANUAL DA SOCIEDADE BRASILEIRA DE ZOOTECNIA, 34., 1997, Juiz de Fora. Anais... Juiz de Fora: Sociedade Brasileira de Zootecnia, 1997. p.293-295.

NATIONAL RESEARCH COUNCIL - NRC. Nutrient requirements of sheep. 6.ed. Washington, D.C.: National Academy Press, 1985. 99p.

NEIVA, J.N.M.; TEIXEIRA, M.; TURCO, S.H.N. et al. Efeito climático sobre parâmetros produtivos e fisiológicos de ovinos Santa Inês mantidos em confinamento na região litorânea do Nordeste do Brasil. Revista Brasileira de Zootecnia, v.33, n.3, p.668-678, 2004.

OLIVEIRA, M.V.M.; PEREZ, J.R.O.; GARCIA, I.F.F. et al. Desempenho de cordeiros das raças Bergamácia e Santa Inês, terminadas em confinamento recebendo dejetos de suínos como parte da dieta. Revista Brasileira de Zootecnia, v.32, n.6, p.1391-1396, 2003.

OREGUI, L.M.; VICENTE, M.S.; GARRO, J. et al. The relationship between body condition score and body weight in Latxa ewes. Options Méditerranéennes - Série Séminaires, n.13, p.109$112,1991$.

OSÓRIO, J.C.S.; OSÓRIO, M.T.M. Produção de carne ovina: técnicas de avaliação in vivo e na carcaça. Pelotas: Universidade Federal de Pelotas, 2003. 73p.

RODRIGUES, M.M.; NEIVA, J.N.; VASCONCELOS, V.R. et al. Utilização do farelo de castanha de caju na terminação de ovinos em confinamento. Revista Brasileira de Zootecnia, v.32, n.1, p.240-248, 2003.

SÁ, J.L.; SIQUEIRA, E.R.; SÁ, C.O. et al. Características de carcaça de cordeiros Hampshire Down e Santa Inês sob diferentes fotoperíodos. Pesquisa Agropecuária Brasileira, v.40, n.3, p.289-297, 2005. 
SIQUEIRA, E.R.; SIMÕES, C.D.; FERNANDES, S. Efeito do sexo e do peso ao abate sobre a produção de carne de cordeiro. I. Velocidade de crescimento, caracteres quantitativos da carcaça, $\mathrm{pH}$ da carne e resultado econômico. Revista Brasileira de Zootecnia, v.30, n3, p.844-848, 2001.

SNOWDER, G.D.; DUCKETT, S.K. Evaluation of the South African Dorper as a terminal sire breed for grown, carcass, and palatability characteristics. Journal of Animal Science, v.81, p.368-375, 2003.

SOUSA W.H., LÔBO, R.N.B.; MORAIS, O.R. Ovinos Santa Inês: estado de arte e perspectivas. In: SIMPÓSIO INTERNACIONAL SOBRE CAPRINOS E OVINOS DE CORTE, 2003, João Pessoa. Anais... SINCORTE, 2003. p.501-522.

SOUZA, P.P.S.; SIQUEIRA, E.R.; MAESTÁ, S.A. Ganho de peso, características de carcaça e dos demais componentes corporais de cordeiros confinados, alimentados com distintos teores de uréia. Ciência Rural, v.34, n.4, p. 1185-1190, 2004.

SUSIN, I.; ROCHA, M.H.M.; PIRES, A.V. et al. Efeito do uso do bagaço de cana-de-açucar in natura ou hidrolizado sobre o desempenho de cordeiros confinados. In: REUNIÃO ANUAL DA SOCIEDADE BRASILEIRA DE ZOOTECNIA, 37, 2000, Viçosa, MG. Anais... Viçosa, MG: Sociedade Brasileira de Zootecnia, 2000. (CD-ROM).
VASCONCELOS, S.H.L.; BRAGA, P.A. RIBEIRO, H.U. et al. Desenvolvimento corporal de ovinos Santa Inês, arraçoados durante o período seco no semi-árido do RN. IIn: REUNIÃO ANUAL DA SOCIEDADE BRASILEIRA DE ZOOTECNIA, 37. 2000, Viçosa, MG. Anais... Viçosa, MG: Sociedade Brasileira de Zootecnia, 2000. (CD-ROM).

VÉRAS, R.M.L.; FERREIRA, M.A.; CAVALCANTI, C.V.A. et al. Substituição do milho por farelo de palma forrageira em dietas para ovinos em crescimento. Desempenho. Revista Brasileira de Zootecnia, v.34, n.1, p.249-256, 2005a.

VÉRAS, R.M.L; FERREIRA, M.A.; VÉRAS, A.S.C. et al. Substituição do milho por farelo de palma forrageira em dietas para ovinos em crescimento. Consumo e digestibilidade. Revista Brasileira de Zootecnia, v.34, n.1, p.351-356, 2005b.

YAMAMOTO, S.M.; MACEDO, F.A.F.; ZUNDT, M. et al. Fontes de óleo vegetal na dieta de cordeiras em confinamento. Revista Brasileira de Zootecnia, v.34, n.2, p.703-710, 2005.

ZUNDT, M; MACEDO, F.A.F.; MARTINS, E.N. et al. Desempenho de cordeiros alimentados com diferentes níveis protéicos. Revista Brasileira de Zootecnia, v.31, n.30, p.1307-1314, 2002. 